\title{
Throwing Motion Control based on Output Zeroing utilizing 2-Link Underactuated Arm
}

\author{
Shunsuke Katsumata, Shigenori Ichinose, Takuya Shoji, Shigeki Nakaura and Mitsuji Sampei \\ Tokyo Institute of Technology, Japan
}

\begin{abstract}
This paper deals with motion control of throwing generated by dexterous action. Dexterous actions can be seen in many sports. In baseball pitching, dexterous throwing seems to use energy transfer and a physical constraint at the elbow joint. To implement the dexterous throwing, two types of twolink underactuated manipulator are presented. One model has a spring at 2nd joint which represents an arm's stiffness and a constraint at elbow joint, another model has a physical absolute constraint at elbow joint. For these models, throwing motion control method based on output zeroing which specifies the path of the ball held by an end-effector is proposed. The proposed control strategy realizes the energy efficient motion for throwing to the desired direction. Simulation and experimental results show the effectiveness of the proposed control method.
\end{abstract}

\section{INTRODUCTION}

We can see dexterous actions in many human sports, e.g. throwing, batting and kicking, etc. In biomechanics field, so many researchers focused on the motion utilizing dexterous action. In addition, there are many researches for motion control based on dexterous action and structure.

As one of such researches for motion control realizing dexterous actions, there is golf swing control whose aim is to perform high-speed swing at the time of hitting ball. Shimojo et al. proposed golf swing motion control applying human dynamic skills[2][3]. They developed two-link golf swing robot that has a constraint around a wrist joint as a dexterous structure. Another work dealt with motion control of swing arm with spring attached to the joint[4]. This work supposed spring attachment as a dexterous structure. Ishikawa et al. studied about batting motion realizing high speed swing[5]. They focused on the sequence of actuation for serial link manipulator. In this paper, we deal with the throwing motion control for another dexterous action.

At first, we deal with features of dexterous action in human throwing. Kreighbaum et al. organized human dexterous action about throw-like motion in biomechanics[1]. They mentioned "Lagging Back" motion that is used to get longer interval for acceleration. After Lagging Back motion, throwing motion is shifted to the motion based on "Kinetic Link Principle". The swing based on the Kinetic Link Principle performs stepwise rotation of joint from proximal link to distal one. This principle is able to realize more efficient motion about energy cost. That movement induces energy flow to gain the velocity of the ball at hand. So we propose the control method for throwing motion based on these principles.

Though there are some researches about motion control for throwing an object, most of them about throwing motion control only dealt with accuracy of the target point to which the released ball approaches[6][7][8]. So, there were few researches about throwing motion control utilizing dexterous features. Ishikawa et al. dealt with throwing motion control that uses energy flow as a dexterous action[9]. But they could not control about the direction of throwing. Our purpose is to implement throwing motion control for desired direction. In addition, we realize the efficient throwing motion from the perspective of energy.

Human throwing is very difficult to analyze all features, because throwing motion is performed in three dimensional space. So we use planar two-link underactuated manipulator as an arm from a lateral view. Two-link manipulator is considered to be enough to realize dexterous features in throwing motion. We suppose that this model has a constraint at the elbow joint for realization of dexterous mechanisms. Two types of model are proposed, one model has a spring at elbow joint, which is represented as a arm's stiffness and a constraint at that joint. We call this model "spring model". Another one has a physical absolute constraint at elbow joint, and we call this model as "constraint model". Motion realization method is based on output zeroing control that specifies the path of the ball held by end-effector.

In this paper, the model, its state equation and throwing pattern are described in section 2. In section 3, we introduce dexterous action which is able to be realized in throwing motion. Motion control scheme is discussed in section 4. Simulation results are given in section 5. In section 6, experimental equipment and results are stated. A conclusion is given in the final section.

\section{THROWING MODEL AND STATE EQUATION}

In throwing motion, we can observe the following phases.

- Wind-up: The arm is swung to the backward direction against the direction to throw. As a result, the distance of movement of hand to accelerate is increased.

- Acceleration: From the position of wind-up, the arm is swung to the direction for throwing with the condition that gains velocity gradually. And then, the ball is released at desired position and velocity.

- Follow Through: From the released position, the arm is slowed down. Finally the arm is stopped fully.

In this paper, we will propose control scheme for the motion from the Acceleration till the Follow Through. So we assume that the initial posture is fixed to the wind-up position stationary. And we also assume that desired direction for throwing is set for horizontal direction (direction of $-x$ axis). 


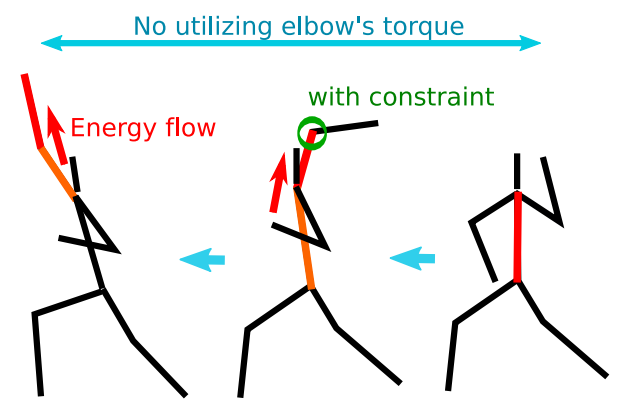

Fig. 1. Dexterous action and structures

To construct throwing model, we briefly state dexterous action, as shown in Fig. 1. Dexterous features are summarized as following three topics:

- Energy transfer from the trunk of the body to the ball in hand is used. This feature enables more energy efficient motion which carries out high speed throwing with fewer energy cost.

- Extension torque at the elbow joint is almost never used in the acceleration phase.

- In the acceleration phase, according to the direction of rotation of the shoulder joint, the arm is swung with constraint at the elbow joint, because the elbow joint has one degree of freedom.

We apply two-link underactuated manipulator as a required minimum model that is possible to express such dexterous features. The proposed model is shown in Fig. 2 and 3. In this paper, we proposed two types of model that are spring model and constraint model.

These models consist of Joint1 as a shoulder joint, Joint2 as an elbow joint, Link1 as an upper arm, and Link2 as a forearm. Joint1 has an actuator, so input torque $\tau$ works only at the Joint1. However Joint 2 has no actuator, so this model is considered as an underactuated manipulator. Underactuated model can represent such dexterous feature that use no extension torque at the elbow joint. TABLE I shows parameter notation for these models.

Fig. 2 shows spring model. This model has a spring attachment at the elbow joint whose spring constant is $K_{c}$. By this spring, this model expresses dexterous structures such as a constraint at elbow and stiffness of the arm. Fig. 3 shows constraint model that represents a physical constraint at elbow as a dexterous structure. This model has a rotating limit to negative direction $\theta_{2 m i n}$.

\section{A. Dynamics of the spring model}

The equation of motion for the spring model can be represented as the follows:

$$
M(q) \ddot{q}+C G(q, \dot{q})=E_{f} \tau,
$$

where $q \in \mathbb{R}^{n}$ is generalized coordinate, $M(q) \in \mathbb{R}^{n \times n}$ is inertia matrix, $C G(q, \dot{q}) \in \mathbb{R}^{n}$ is Coriolis and centrifugal force and gravity term, and $E_{f}=[1,0]^{T}$. Then, the state equation for the spring model is represented by

$$
x=f(x)+g(x) \tau,
$$

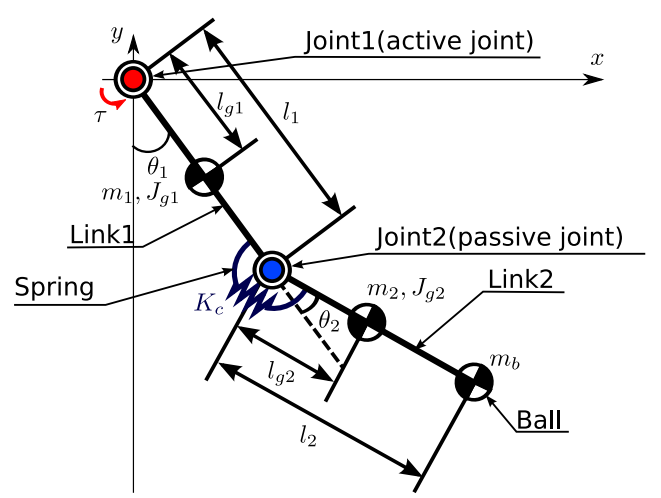

Fig. 2. Spring model

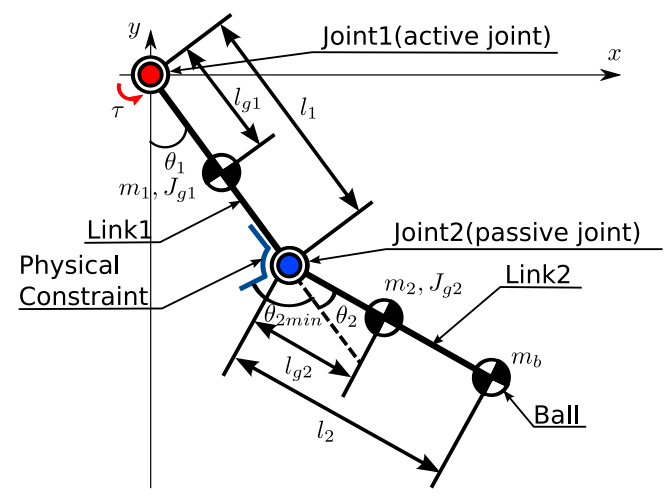

Fig. 3. Constraint model

TABLE I

PARAMETER NOTATION

\begin{tabular}{c|l}
\hline$J_{g i}$ & moment of inertia of the $i$-th link \\
$l_{i}$ & length of the $i$-th link \\
$l_{g i}$ & length from the $i$-th joint to COG of the $i$-th link \\
$m_{i}$ & mass of the $i$-th link \\
$m_{b}$ & mass of the ball \\
$K_{c}$ & spring constant \\
$\theta_{2 m i n}$ & constraint angle of the 2nd joint \\
\hline
\end{tabular}

where

$$
f(x)=\left[\begin{array}{c}
\dot{q} \\
-M(q)^{-1} C G(q, \dot{q})
\end{array}\right], \quad g(x)=\left[\begin{array}{c}
0 \\
M(q)^{-1} E_{f}
\end{array}\right],
$$

with $x=\left[q^{T}, \dot{q}^{T}\right]^{T}$. This equation has the term $K_{c}\left(\theta_{2}-\theta_{k n}\right)$ in $C G_{2}(q, \dot{q})$ of $C G(q, \dot{q})=\left[C G_{1}(q, \dot{q}), C G_{2}(q, \dot{q})\right]^{T}$. This is the term due to spring force and $\theta_{k n}$ is a natural angle of spring.

\section{B. Dynamics of the constraint model}

The equation of motion for the constraint model can be represented as follows:

$$
\begin{aligned}
M(q) \ddot{q}+C G(q, \dot{q}) & =E_{f} \tau+J^{T} \lambda, \\
J \dot{q} & =0,
\end{aligned}
$$

where $J \in \mathbb{R}^{n}$ is the Jacobian. $J$ is represented as

$$
J=\frac{\partial N}{\partial q}
$$


where $N=\theta_{2}$ is constraint state. When Link2 reaches to constraint limit angle $\theta_{2 \mathrm{~min}}$, constraint torque $\lambda$ occurs around $\theta_{2}$. And $\lambda$ is represented as

$$
\lambda=\left(J M^{-1} J^{T}\right)^{-1} J M^{-1}\left(C G(q, \dot{q})-E_{f} \tau\right) .
$$

Then, the state equation for the constraint model is represented by

$$
x=f(x)+g_{u}(x) \tau+g_{f}(x) \lambda,
$$

where

$$
\begin{aligned}
f(x) & =\left[\begin{array}{c}
\dot{q} \\
-M(q)^{-1} C G(q, \dot{q})
\end{array}\right], g_{u}(x)=\left[\begin{array}{c}
0 \\
M(q)^{-1} E_{f}
\end{array}\right], \\
g_{f}(x) & =\left[\begin{array}{c}
0 \\
M(q)^{-1} J^{T}
\end{array}\right] .
\end{aligned}
$$

\section{ENERGY FLOW OF DEXTEROUS ACTIONS}

In this section, we analyze energy transfer which is one of the factor of the dexterous action in human throwing. This feature can be applied to either spring model and constraint model. To apply this feature to both models, we suppose that Joint 2 doesn't have any constraint in the following discussion about energy.

First, energy of the Link1 $E_{1}$, energy of the Link2 $E_{2}$, energy of the ball $E_{b}$ and total energy of the system $E$ are given by

$$
\begin{aligned}
E_{1} & =\frac{1}{2} m_{1}\left(\dot{x}_{1}^{2}+\dot{y}_{1}^{2}\right)+\frac{1}{2} J_{g 1} \dot{\theta}_{1}^{2}+m_{1} g\left(y_{1}+l_{1}+l_{2}\right) \\
E_{2} & =\frac{1}{2} m_{2}\left(\dot{x}_{2}^{2}+\dot{y}_{2}^{2}\right)+\frac{1}{2} J_{g 2}\left(\dot{\theta}_{1}+\dot{\theta}_{2}\right)^{2}+m_{2} g\left(y_{2}+l_{1}+l_{2}\right) \\
E_{b} & =\frac{1}{2} m_{b}\left(\dot{x}_{b}^{2}+\dot{y}_{b}^{2}\right)+m_{b} g\left(y_{b}+l_{1}+l_{2}\right) \\
E & =E_{1}+E_{2}+E_{b}
\end{aligned}
$$

where $\left(x_{1}, y_{1}\right),\left(x_{2}, y_{2}\right)$ are position of the mass of the Link1 and the Link 2 in Cartesian coordinate, and $\left(x_{b}, y_{b}\right)$ is position of the mass of the ball. Velocity of the ball and its time derivative are given by

$$
\begin{aligned}
& v_{b}=\sqrt{\dot{x}_{b}^{2}+\dot{y}_{b}^{2}}, \\
& \dot{v}_{b}=\frac{1}{m_{b} v_{b}}\left[\dot{E}_{b}+m_{b} g \dot{y}_{b}\right] .
\end{aligned}
$$

According to (13), it can be said that the velocity of the ball is increased, if the time derivative of the energy of the ball $\dot{E}_{b}$ is increased. In addition, $\dot{E}_{b}$ is represented as

$$
\dot{E}_{b}=\tau \dot{\theta}_{1}-\left(\dot{E}_{1}+\dot{E}_{2}\right) \text {. }
$$

Equation (14) denotes that the energy of the ball can be accumulated by transferring the energy from the Link1 and Link2 to the ball. As a result, according to (13) and (14), the velocity of the ball can be gained by realizing the motion so as to transfer the energy of the link to the energy of the ball.

The time derivative of the energy of the ball $\dot{E}_{b}$ is also represented as the following equation, when gravity term is eliminated.

$$
\dot{E}_{b}=\frac{l_{1} m_{b}}{\Delta}\left[\tau H_{\tau}+H_{1}+H_{2}\right]
$$

where

$$
\begin{aligned}
& H_{\tau}=w_{\tau 1} \dot{\theta}_{1}+w_{\tau 2} \dot{\theta}_{2} \\
& H_{1}=2 \sin \theta_{2}\left(w_{11} \dot{\theta}_{1}+w_{12} \dot{\theta}_{2}\right) \dot{\theta}_{1}\left(\dot{\theta}_{1}+\dot{\theta}_{2}\right) \\
& H_{2}=l_{1}\left(l_{g 2} m_{2}+l_{2} m_{b}\right) \sin 2 \theta_{2}\left(w_{21}\left(\dot{\theta}_{1}+\dot{\theta}_{2}\right)^{3}+w_{22} \dot{\theta}_{1}^{3}\right)
\end{aligned}
$$

$$
\begin{aligned}
w_{\tau 1}= & l_{1}\left[2 J_{g 2}-\left(l_{2}-2 l_{g 2}\right) l_{g 2} m_{2}+l_{2}^{2} m_{b}\right]-l_{1} l_{2}\left(l_{g 2} m_{2}+l_{2} m_{b}\right) \\
& \cos 2 \theta_{2}+2 l_{2}\left[J_{g 2}+l_{g 2}\left(-l_{2}+l_{g 2}\right) m_{2}\right] \cos \theta_{2} \\
w_{\tau 2}= & 2 l_{2}\left[J_{g 2}+l_{g 2}\left(-l_{2}+l_{g 2}\right) m_{2}\right] \cos \theta_{2} \\
w_{11}= & \left\{-l_{2}^{2}\left(J_{g 1}+l_{g 1}^{2} m_{1}\right)+l_{1}^{2}\left[J_{g 2}+\left(-l_{2}^{2}+l_{g 2}^{2}\right) m_{2}\right]\right\}\left(l_{g 2} m_{2}+\right. \\
& \left.l_{2} m_{b}\right) \\
w_{12}= & -\left\{J_{g 1} l_{2}+l_{2} l_{g 1}^{2} m_{1}+l_{1}^{2}\left(l_{2}-l_{g 2}\right) m_{2}\right\}\left(J_{g 2}\right. \\
& \left.+l_{g 2}^{2} m_{2}+l_{2}^{2} m_{b}\right) \\
w_{21}= & l_{2}\left[J_{g 2}+\left(-l_{2}+l_{g 2}\right) l_{g 2} m_{2}\right] \\
w_{22}= & -\left[l_{2}\left(J_{g 1}+l_{g 1}^{2} m_{1}\right)+l_{1}^{2}\left(l_{2}-l_{g 2}\right) m_{2}\right] \\
\Delta= & \left(J_{g 2}+l_{g 2}^{2} m_{2}+l_{2}^{2} m_{b}\right)\left(J_{g 1}+l_{g 1}^{2} m_{1}+l_{1}^{2}\left(m_{2}+m_{b}\right)\right) \\
& -l_{1}^{2}\left(l_{g 2} m_{2}+l_{2} m_{b}\right)^{2} \cos ^{2} \theta_{2}
\end{aligned}
$$

Next, we show the condition that the time derivative of the energy of the ball is increased $\left(\dot{E}_{b}>0\right)$. It can be divided into two cases relating to the direction of the whole energy flow, one is the situation where the total energy of the system is accumulated $\left(\tau \dot{\theta}_{1}>0\right)$, another one is the situation that the total energy of the system is dissipated $\left(\tau \dot{\theta}_{1}<0\right)$.

\section{A. The case of increasing the total energy}

If following assumptions are satisfied, $\dot{E}_{b}$ is increased in the case of increasing the total energy.

- $\dot{\theta}_{1}>0, \dot{\theta}_{2} \geq 0$

- $\tau>0$

- $-\pi \leq \theta_{2} \leq \theta_{2 \tau}$

- $\left|w_{\tau 1} \dot{\theta}_{1}\right|>\left|w_{\tau 2} \dot{\theta}_{2}\right|$

where $\theta_{2 \tau}$ is the angle when $w_{\tau 1}=0\left(-\pi \leq \theta_{2} \leq 0\right)$. These conditions are the situation increasing the total energy of the system by input $\tau$, because $\tau \dot{\theta}_{1}>0 . H_{\tau}>0, H_{1}>0$ and $H_{2}>0$ should be satisfied to be $\dot{E}_{b}>0$.

Generally, it is clear that $H_{1}>0$ and $H_{2}>0$ from (16b) and $(16 \mathrm{c})$ when $\dot{\theta}_{1}>0$ and $\dot{\theta}_{2}>0$, because $w_{11}, w_{12}, w_{21}$ and $w_{22}$ consist only of physical parameters.

Next, we think about whether $H_{\tau}>0$ is satisfied or not. If $-\pi \leq \theta_{2} \leq \theta_{2 \tau}$, then it can be said that $w_{\tau 1}>0$. And it is clear that $w_{\tau 2}<0$ from (17b) $\left(-\pi \leq \theta_{2} \leq 0\right)$. Therefore, it is said that $H_{\tau}>0$, when $\left|w_{\tau 1} \dot{\theta}_{1}\right|>\left|w_{\tau 2} \dot{\theta}_{2}\right|$ is satisfied. This assumption is fulfilled in the case of $\dot{\theta}_{1} \gg \dot{\theta}_{2}$.

For example, the image of this situation can be represented as in Fig. 4. The arm is folded back at initial posture as $\theta_{2}<0$ in this situation, and then it is accelerated as $\dot{\theta}_{1}>0$. Thus, the energy of the ball can be increased in the case of increasing the total energy of the system, when the assumptions described above are satisfied. 


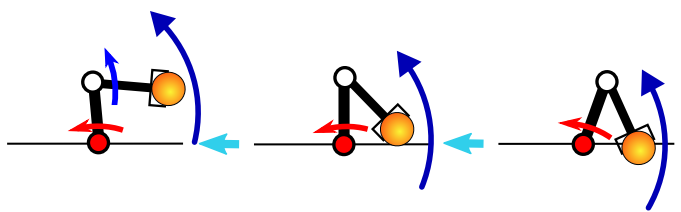

Fig. 4. The motion that occurs energy transfer (In the case of increasing the total energy)

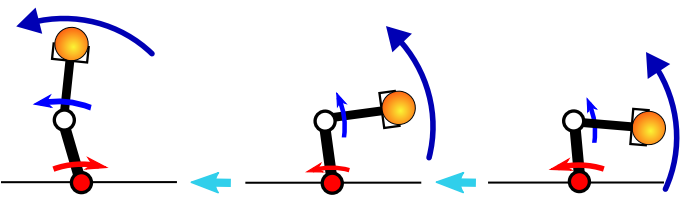

Fig. 5. The motion that occurs energy transfer (In the case of decreasing the total energy)

\section{B. The case of decreasing the total energy}

If following assumptions are satisfied, $\dot{E}_{b}$ is increased in the case of decreasing the total energy.

- $\dot{\theta}_{1}>0, \dot{\theta}_{2} \geq 0$

- $\tau<0$

- $-\frac{\pi}{2} \leq \theta_{2} \leq 0$

- $\left|w_{\tau 1} \dot{\theta}_{1}\right|<\left|w_{\tau 2} \dot{\theta}_{2}\right|$

In these assumptions the different points from the case of increasing the total energy are $\tau<0$ and $\left|w_{\tau 1} \dot{\theta}_{1}\right|<\left|w_{\tau 2} \dot{\theta}_{2}\right|$.

$H_{\tau}<0, H_{1}>0$ and $H_{2}>0$ should be satisfied to be $\dot{E}_{b}>0$. $H_{1}>0, H_{2}>0$ can be satisfied from the same reason denoted in the case of increasing the total energy. To think about about $H_{\tau}<0$, first $w_{\tau 2}<0$ is satisfied in $-\frac{\pi}{2} \leq \theta_{2} \leq 0$. Second, considering for $w_{\tau 1}$, it is switched from positive to negative at $\theta_{2 \tau}$, when $\theta_{2}$ increases from $-\frac{\pi}{2}$ to 0 . So it can be said that $H_{\tau}>0$, if $\left|w_{\tau 1} \dot{\theta}_{1}\right|<\left|w_{\tau 2} \dot{\theta}_{2}\right|$ is satisfied. This assumption is satisfied in the case of $\dot{\theta}_{1} \ll \dot{\theta}_{2}$.

For example, the image of this situation can be represented as in Fig. 5. In this figure, we think about the movement after the motion performed in the case of increasing the total energy (Fig. 4). If $\tau$ is switched from positive to negative after the arm is sufficiently accelerated as $\dot{\theta}_{1}>0$, energy transfer occurs from the link to the ball. As a result, the arm is swung like Link1 is decelerated by input torque $\tau<0$.

\section{CONTROL METHOD OF THROWING MOTION}

In this section, we explain a control method to throw the ball toward the desired direction using underactuated model described in $\S I I$. There are mainly two objectives:

- Execution of throwing in the desired direction (i.e. horizontal direction).

- Realization of energy efficient throwing by using the energy transfer from the link to the ball (stated in §III).

However, this model has one actuator at Joint1 as a shoulder, so we can not make the ball track the desired trajectory. In other words, we can't make the ball the desired state which includes position and velocity. To throw in the desired direction, we will control the position of the ball to follow a straight path. If the ball is accelerated along this straight path, it can be thrown in the desired direction. Additionally, the energy transfer described in $\S \mathrm{III}$ will occur by realizing acceleration of the ball on that path, because the conditions that increase the time derivative of the energy of the ball can be satisfied when the ball at end-effector moves along the straight path.

We propose the control method for throwing motion in each model, i.e. spring model and constraint model as follows. Note that throwing motion control of spring model should not be compared with that of constraint model for the reasons that these models deal with different features respectively. For example, spring model uses the effect of spring, but constraint model only uses the effect of physical constraint. Therefore we denote the advantage of each model by using each features.

\section{A. The case of the spring model}

The desired path for throwing is chosen as

$$
y_{b}=-r_{d 2}+\frac{2 r_{d 2}}{1+\exp \left[\alpha\left(x_{b}-r_{d 1}\right)\right]},
$$

where $\left(x_{b}, y_{b}\right)$ is the position of the ball, and $r_{d 1}, r_{d 2}, \alpha$ are parameters for the desired path. Equation (18) is drawn in Fig. 6. $r_{d 1}$ is initial position of the ball in x-axis, $r_{d 2}$ is distance between straight line of the desired path and $\mathrm{x}$-axis, and $\alpha$ represents gradient of a curve section. Thus these parameters of this desired path are easy to understand. Note that the desired path is connected smoothly between the curve section and the straight section. If the ball moves from the curve section of the desired path to the straight section of it (Fig. 7), then the ball can be released to desired direction after the ball velocity reaches the target velocity for release.

To make the ball converge to the desired path, we use output zeroing control[10]. The output function for zeroing is set as following.

$$
h=y_{b}+r_{d 2}-\frac{2 r_{d 2}}{1+\exp \left[\alpha\left(x_{b}-r_{d 1}\right)\right]}
$$

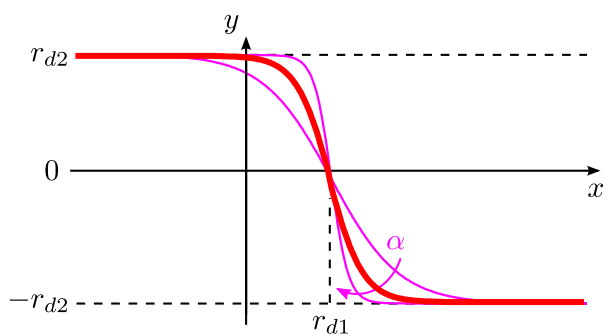

Fig. 6. Desired path

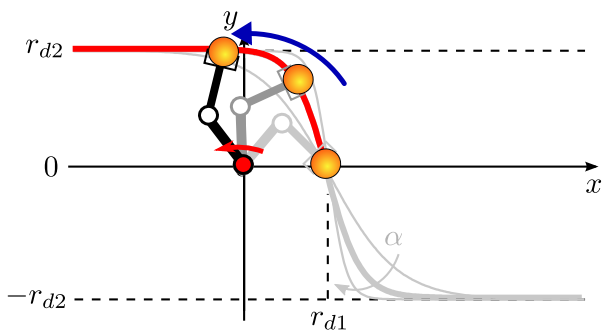

Fig. 7. Desired motion (Spring model) 


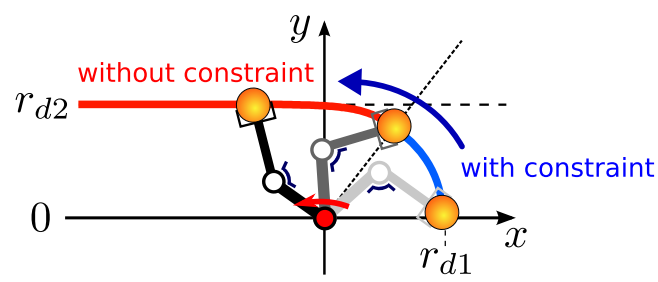

Fig. 8. Desired motion (Constraint model)

Then, $\dot{h}, \ddot{h}$ are calculated as follows:

$$
\begin{aligned}
\dot{h} & =\frac{\partial h}{\partial x}(f(x)+g(x) u) \\
& =L_{f} h+u L_{g} h=L_{f} h, \\
\ddot{h} & =\frac{d L_{f} h}{d t} \\
& =L_{f}^{2} h+u L_{g} L_{f} h .
\end{aligned}
$$

where $L_{f} h, L_{g} h, L_{f}^{2} h$ and $L_{g} L_{f} h$ are Lie derivative[10]. By taking new input as $\ddot{h}=v$, the input for output zeroing is obtained as the following form.

$$
u=L_{g} L_{f} h^{-1}\left[v-L_{f}^{2} h\right] .
$$

If the new input $v$ can stabilize the new state $[h, \dot{h}]$, the ball at the end-effector will converge on the desired path for throwing. This means output zeroing is achieved. Additionally, if zero dynamics that makes a movement on the desired path is unstable by the effect of the spring, the ball is accelerated on the desired path. Thus, we carry out throwing motion control for the desired direction using output zeroing.

\section{B. The case of the constraint model}

In the case of the constraint model, we make the following assumption for initial state.

- The initial angle $\theta_{20}$ equals to the constraint angle $\theta_{2 \min }$. Thus, throwing motion is started from constraint condition. When Joint 2 is constrained, the trajectory of the ball must be a circular arc whose center locates on Joint1. Therefore, two types of controller are used in each case:

- With constraint phase: The input torque is constant $u_{c}$, while the state is constrained from the initial state.

- Without constraint phase: The input is derived using output zeroing. The output function for zeroing is (19).

Fig. 8 shows the movement described above. At the timing of switching the phase, the parameters of the output function (19) are selected to connect the trajectory smoothly by calculating curvature of the desired path. So parameters of output function are selected by initial angle $\theta_{20}$ and the position of breaking constraint condition.

\section{SIMULATIONS}

In this section, we verify effectiveness of the proposed control method for throwing through numerical simulations. Physical parameters used in simulation are shown in TABLE II, but $K_{c}=0.0[\mathrm{Nm} / \mathrm{rad}]$ in the case of the constraint model

\begin{tabular}{|c|c|c|c|c|c|}
\hline \multicolumn{6}{|c|}{ PHYSICAL PARAMETERS } \\
\hline$m_{1}$ & $2.0[\mathrm{~kg}]$ & $l_{1}$ & $0.40[\mathrm{~m}]$ & $J_{g 1}$ & $\frac{m_{1} l_{1}^{2}}{12}$ \\
\hline$m_{2}$ & $1.8[\mathrm{~kg}]$ & $l_{2}$ & $0.48[\mathrm{~m}]$ & $J_{g 2}$ & $\frac{m_{2} l_{2}^{2}}{12}$ \\
\hline$m_{b}$ & $0.1[\mathrm{~kg}]$ & $l_{g 1}$ & $0.20[\mathrm{~m}]$ & $\theta_{k n}$ & $0.0[\mathrm{deg}]$ \\
\hline$K_{c}$ & $6.0[\mathrm{Nm} / \mathrm{rad}]$ & $l_{g 2}$ & $0.24[\mathrm{~m}]$ & & \\
\hline
\end{tabular}
and also rotational limit $\theta_{2 \min }$ is applied only in the case of constraint model. We show each result about the spring model and the constraint model.
TABLE II

We suppose that the ball is released after the releasing requirement is satisfied. The releasing requirement is defined as

$$
U_{\text {rel }}:=\left\{q_{b}\left|v_{b} \geq v_{r e l},\right| \dot{y}_{b} \mid<\dot{y}_{r e l}, c_{b}<c_{r e l}\right\}
$$

where $q_{b}=\left[x_{b}, y_{b}, \dot{x}_{b}, \dot{y}_{b}\right]^{T}$ is state of the ball. $c_{b}$ is curvature of the ball trajectory. $v_{r e l}$ is desired velocity of the ball for release. $\dot{y}_{r e l}$ is a restriction to throw the ball to desired direction( $-x$-axis). $c_{r e l}$ is a restriction to verify whether the trajectory of the ball can be kept on straight path for throwing. If above requirements are satisfied, the end-effector will release the ball.

\section{A. The case of the spring model}

To select the parameters of the output function, we calculate optimal value of these parameters utilizing genetic algorithm which searches the parameters of the output function minimizing the value of the energy $\operatorname{cost} E_{c}$. Here $E_{c}$ is represented as

$$
E_{c}=\int_{0}^{T_{r e l}}\left|\tau \dot{\theta}_{1}\right| d t,
$$

where $T_{r e l}$ is the timing of release.

Control parameters for the spring model and the optimized parameters are shown in TABLE III. And we define $E_{c v}$ as

$$
E_{c v}=\frac{\frac{m_{b} v_{b}^{2}}{2}}{\int\left|\tau \dot{\theta}_{1}\right| d t} .
$$

$E_{c v}$ is an evaluation value to verify energy efficiency of throwing motion. Namely $E_{c v}$ indicates contribution ratio of the kinetic energy of the ball against the energy cost. If $E_{c v}$ takes a high value, we can consider that the inflow energy by input torque is spent to accelerate the ball efficiently.

Simulation results are shown in Fig. 9. $E_{c}$ is $92.56[\mathrm{~J}]$ with released velocity $15.00[\mathrm{~m} / \mathrm{s}]$. Fig. 9(a) shows that the trajectory of the ball $\left(x_{b}, y_{b}\right)$ and the trajectory of the endeffector $\left(x_{\text {end }}, y_{\text {end }}\right)$. Before releasing, $\left(x_{b}, y_{b}\right)$ equals to $\left(x_{\text {end }}\right.$, $\left.y_{\text {end }}\right)$. It can be seen that throwing motion in desired direction is realized. The velocity of the ball $v_{b}$ is shown in Fig. 9(b). $v_{b}$ is increased during the acceleration motion, and it reaches to the desired velocity for release.

Fig. 9(c) shows angular velocity $\dot{\theta}_{1}, \dot{\theta}_{2}$ and Fig. 9(d) shows input torque $\tau$. After $t=0.2[\mathrm{~s}], \dot{\theta}_{2}$ is rapidly increased against $\dot{\theta}_{1}$ is decreased. And $\tau$ has a peak at $t=0.183[\mathrm{~s}]$, and then it is decreased. From these transition of $\dot{\theta}_{1}, \dot{\theta}_{2}$ and $\tau$, it can be said that this throwing motion uses energy transfer from the link to the ball to accelerate the velocity of the ball efficiently. The energy of links and ball $E_{1}, E_{2}$ and $E_{b}$ are shown in Fig. 9(e). In this figure, it also can be seen that the energy transfer is caused from the point where $E_{b}$ is increased against $E_{1}, E_{2}$ is decreased. 
TABLE III

CONTROL PARAMETERS (SPRING MODEL)

\begin{tabular}{|c|c||c|l||l|l|}
\hline$\theta_{10}$ & $140.10[\mathrm{deg}]$ & $\alpha$ & 5.8 & $v_{r e l}$ & $15.0[\mathrm{~m} / \mathrm{s}]$ \\
$\theta_{20}$ & $-89.78[\mathrm{deg}]$ & $r_{d 1}$ & 0.626 & $\dot{y}_{r e l}$ & $0.5[\mathrm{~m} / \mathrm{s}]$ \\
& & $r_{d 2}$ & 0.751 & $c_{r e l}$ & 0.3 \\
\hline
\end{tabular}
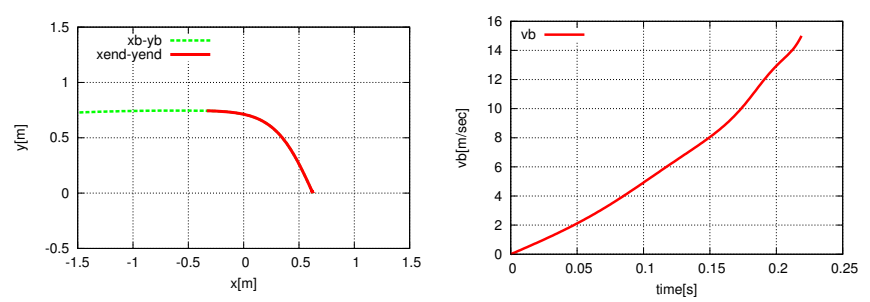

(a) Trajectory of the ball : $x_{b}, y_{b}$

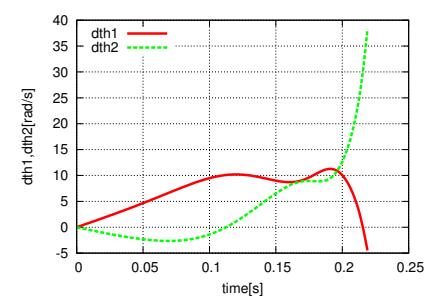

(c) Angular velocity : $\dot{\theta}_{1}, \dot{\theta}_{2}$

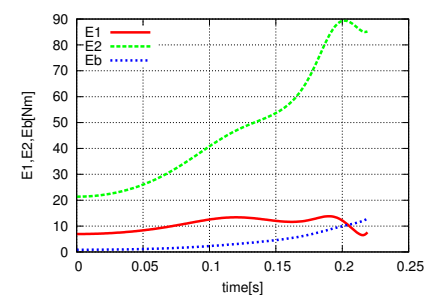

(e) Energy : $E_{1}, E_{2}, E_{b}$

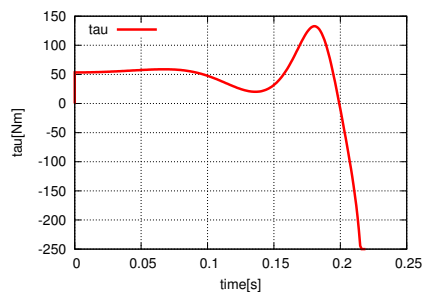

(d) Input torque : $\tau$

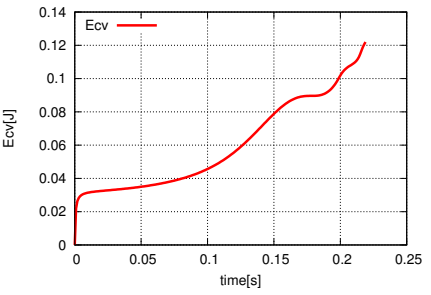

(f) $E_{c v}$

Fig. 9. Throwing simulation (Spring model)

Fig. 9(f) shows the evaluation value $E_{c v}$ defined by (25). $E_{c v}$ is increased till release. Therefore this control method is efficient from the perspective of energy.

Since these simulation results are optimized about energy cost, the parameters of the desired path is selected to use the effect of spring positively. From Fig. 9(d), $\tau$ is decreased once after $t=0.1$ [sec], and then it is increased. This decrease of torque occurs from using potential energy of spring instead of the input torque. Besides, optimized parameter has the relation that $r_{d 2}$ is larger than $r_{d 1}$. By using these parameters, $\dot{\theta}_{2}$ is increased until the ball enters to the straight section of desired path. As a result, potential energy of the spring is used to accelerate the ball.

\section{B. The case of the constraint model}

We also carry out the simulation of throwing motion control using constraint model. Control parameters are shown in TABLE IV. Fig. 10 shows simulation results, $E_{c}=105.46[\mathrm{~J}]$ with released velocity $13.20[\mathrm{~m} / \mathrm{s}]$ at $t=0.274[\mathrm{~s}]$.

Fig. 10(a) shows the trajectory of $\left(x_{b}, y_{b}\right)$ and $\left(x_{\text {end }}, y_{\text {end }}\right)$. It can be seen that the ball is released on the straight path, and we can say that throwing for desired direction is realized.
TABLE IV

CONTROL PARAMETERS (CONSTRAINT MODEL)

\begin{tabular}{|c|l||c|l||l|l|}
\hline$\theta_{10}$ & $-140.20[\mathrm{deg}]$ & $\alpha$ & 5.54 & $v_{\text {rel }}$ & $10.0[\mathrm{~m} / \mathrm{s}]$ \\
$\theta_{20}$ & $-90.00[\mathrm{deg}]$ & $r_{d 1}$ & 0.625 & $\dot{y}_{r e l}$ & $0.5[\mathrm{~m} / \mathrm{s}]$ \\
$u_{c}$ & $40.0[\mathrm{Nm}]$ & $r_{d 2}$ & 0.680 & $c_{r e l}$ & 0.3 \\
\hline
\end{tabular}

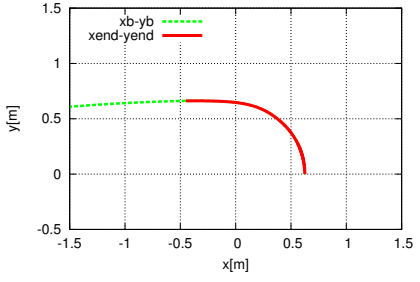

(a) Trajectory of the ball : $x_{b}, y_{b}$

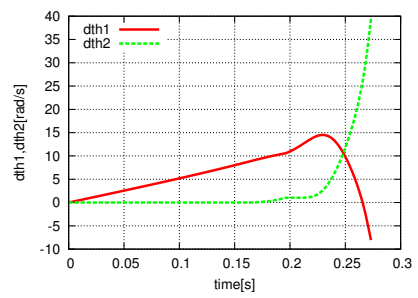

(c) Angular velocity : $\dot{\theta}_{1}, \dot{\theta}_{2}$

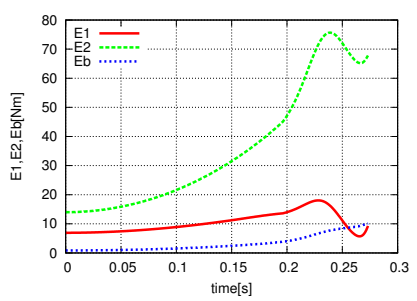

(e) Energy : $E_{1}, E_{2}, E_{b}$

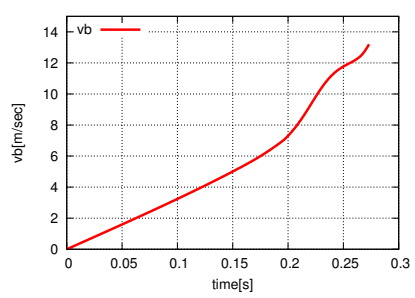

(b) Ball velocity : $v_{b}$

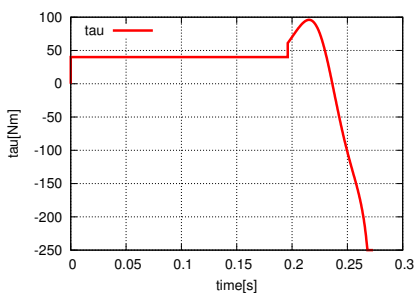

(d) Input torque : $\tau$

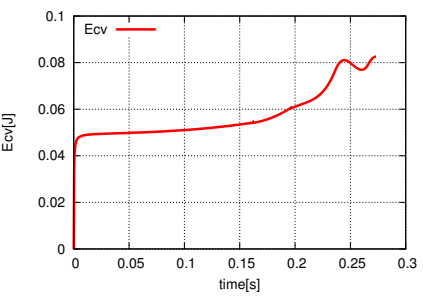

(f) $E_{c v}$
Fig. 10. Throwing simulation (Constraint model)

Fig. 10(b) shows the ball velocity $v_{b}$. From this figure, it is clear that $v_{b}$ is rapidly increased on the straight path.

From the same reason denoted in the simulation results of spring model, it can be said that the energy of the ball is increased by energy transfer as in Fig. 10(e). And we can see in Fig. 10(c),(d) that the condition described in $\S I I I$ is satisfied which increases the energy of the ball.

Fig. 10(e) shows $E_{c v}$ that is the value for evaluation. It can be seen that the value of $E_{c v}$ is rapidly increased after $t=$ 0.2 [s], so the acceleration of the ball is performed efficiently about energy. Fig. 11 shows a snapshot of the simulation of throwing motion in the case of the constraint model.

1) Comparing the minimum energy cost: We compare with another model to confirm the advantage of proposed model. Here, we compare minimum energy cost for the constraint model with the one for 1-link model. 1-link model can be considered that it doesn't rotate around Joint2 $\left(\dot{\theta}_{2}=\right.$ $0)$. Input limit is set as $U_{\max }=50.0[\mathrm{Nm}], U_{\min }=-50.0[\mathrm{Nm}]$. After the ball is released, the minimum energy cost is calculated for each initial state, Fig. 12 shows simulation results for each initial state $\theta_{20}$. Once initial angle $\theta_{20}$ is selected, all initial states are fixed, because initial position 


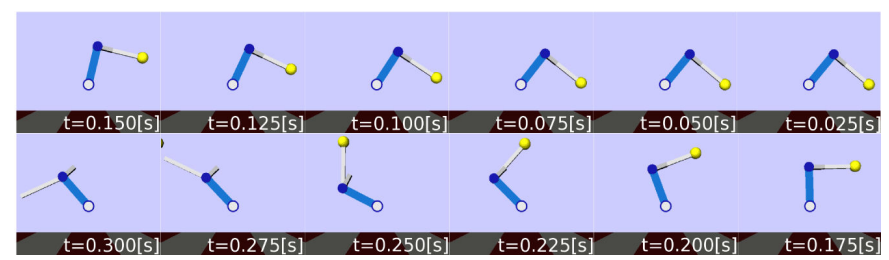

Fig. 11. Sequences of throwing motion (Constraint model) (a) Released velocity

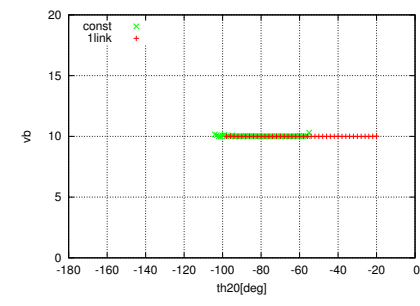

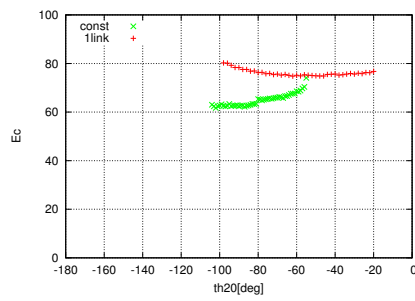

(b) Energy cost : $E_{c}$
Fig. 12. Minimum energy cost

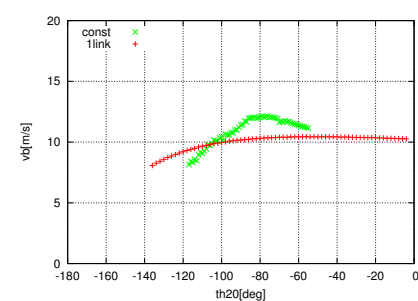

(a) Released velocity

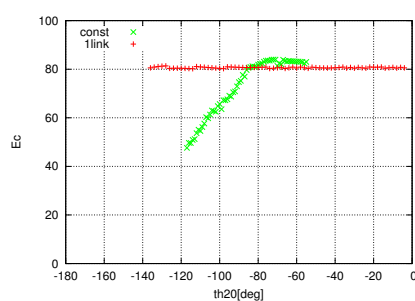

(b) Energy cost : $E_{c}$
Fig. 13. Maximum released velocity

of the ball at the end-effector is located on the x-axis by assumption.

Fig. 12(a) shows the velocity of the ball and Fig. 12(b) shows energy cost $E_{c}$. It can be seen that proposed method is less minimum $E_{c}$ than 1-link model, when throwing is performed at same released velocity. However, the range which $\theta_{20}$ satisfies requirement of release is restricted, because input torque is limited.

2) Comparing the maximum ball velocity: By using proposed control method, we compare maximum velocity of the ball at the moment of release for the constraint model with the one for 1-link model. The maximum ball velocity is calculated at each initial angle $\theta_{20}$, and input limit is set as $U_{\max }=50.0[\mathrm{Nm}]$ and $U_{\min }=-50.0[\mathrm{Nm}]$.

Fig. 13(a) shows the velocity of the ball and Fig. 13(b) shows energy cost $E_{c}$. From Fig. 13(a), it can be said that proposed method can realize higher velocity of the ball at the release than the case of the 1-link model. Besides, we take notice of the range between $\theta_{20}=-110[\mathrm{deg}]$ and $\theta_{20}=-85[\mathrm{deg}]$, then throwing using the proposed method performs higher velocity of the released ball with fewer energy cost comparing the case of the 1-link model.

By using proposed model, which is an underactuated model with constraint at Joint2, we realized throwing the ball to the desired direction through numerical simulations. In addition, from the results of comparing with 1-link model, proposed control method is more efficient from the viewpoint of energy cost than 1-link model.

\section{EXPERIMENT}

In this section, we state experiment of throwing motion to verify the validity of our model and the effectiveness of proposed control method. Note that experimental results described here are still in progress.

\section{A. Experimental equipment}

We developed an experimental equipment for throwing motion as shown in Fig.14-16. Fig. 14 shows overview of the experimental equipment. Physical parameters of this equipment are listed in TABLE $\mathrm{V}$ where $d_{i}$ is a viscous friction of $i$-th joint. Although these parameters are different in TABLE II, the objective in this section is to confirm not simulation results discussed previous section but our approach for throwing motion control in this paper.

This experimental equipment is constructed as the spring model Tennis ball is used for a throwing object. Joint1 is driven by an AC servo motor. Joint2 has no actuator, but torsion coil spring is attached as a constraint. This structure can be seen in Fig.15. As the proposed model is underactuated model, this equipment can't be fixed at the Wind-up position that is initial position. Therefore Joint 2 of experimental equipment has an electromagnet to fix Joint2, and electromagnetic force is applied at the initial position. Link2 is shown in Fig.16. It can be seen that the hand mechanism to hold the ball consists of an electromagnet and a compression spring. It holds the ball when the compression spring is shortened by electromagnetic force, and it can release the ball instantly to turn off the electromagnet.

\section{B. Experimental results}

We carried out experiment of throwing motion control. Control parameters are listed in TABLE VI. Experimental results are shown in Fig. 17. Note that experimental result and simulation result which is carried out by using parameters in TABLE V and VI are drawing in these figures.

Fig. 17(a) shows the trajectory of the ball. From this figure, it can be seen that throwing for desired direction is performed in experiment. Fig.17(b) shows the velocity of the ball which is increased until release. Angular velocity $\dot{\theta}_{1}, \dot{\theta}_{2}$ are shown in Fig.17(c). Fig. 17(d) shows input torque $\tau$ that is limited by $U_{\text {max }}=79[\mathrm{Nm}], U_{\text {min }}=-79[\mathrm{Nm}]$. The energy of link $E_{1}$, $E_{2}$ and the energy of the ball $E_{b}$ are shown in Fig. 17(e). Energy transfer from the link to the ball can be seen in this figure. Fig. 17(f) shows $E_{c v}$ defined as (25). This result shows that input energy is spent efficiently to accelerate the ball, because $E_{c v}$ is increased rapidly.

\section{CONCLUSIONS}

In this paper, we discussed throwing motion control as dexterous action based on output zeroing control utilizing 2-link underactuated arm.

First, we introduced dexterous actions in throwing motion, and two types of underactuated model that are the spring model and the constraint model was proposed. 


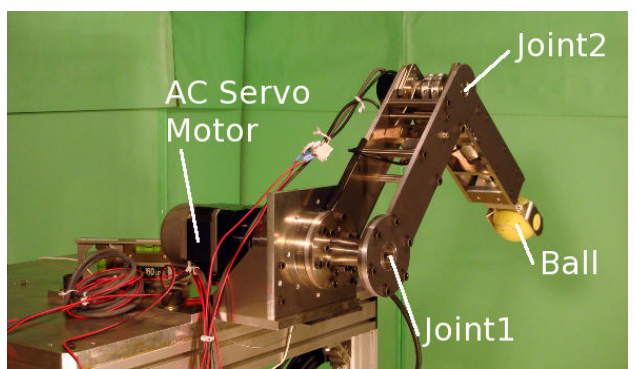

Fig. 14. Overview of the experimental equipment

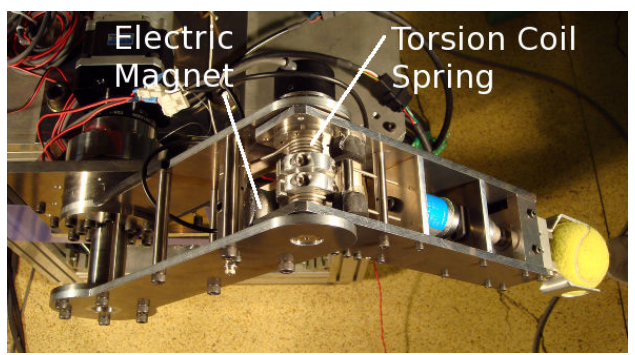

Fig. 15. Magnification around joint2

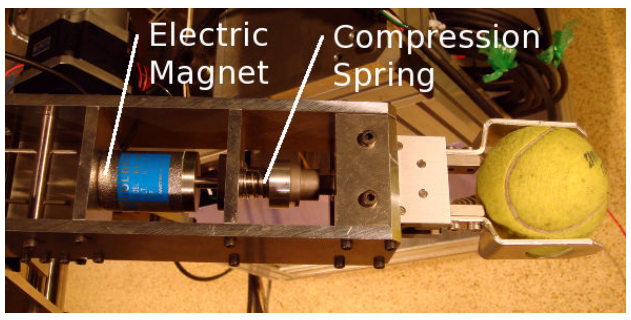

Fig. 16. The release mechanism in link2

TABLE V

PHYSICAL PARAMETERS (EXPERIMENT)

\begin{tabular}{|c|l||c|l|}
\hline$m_{1}$ & $1.972[\mathrm{~kg}]$ & $d_{1}$ & $0.4847\left[\mathrm{kgm}^{2}\right]$ \\
$m_{2}$ & $1.537[\mathrm{~kg}]$ & $d_{2}$ & $0.0432\left[\mathrm{kgm}^{2}\right]$ \\
$m_{b}$ & $0.057[\mathrm{~kg}]$ & $J_{g 1}$ & $0.0559\left[\mathrm{kgm}^{2}\right]$ \\
$l_{1}$ & $0.25[\mathrm{~m}]$ & $J_{g 2}$ & $0.0206\left[\mathrm{kgm}^{2}\right]$ \\
$l_{2}$ & $0.30[\mathrm{~m}]$ & $\theta_{k n}$ & $0.0[\mathrm{deg}]$ \\
$l_{g 1}$ & $0.0929[\mathrm{~m}]$ & $K_{c}$ & $1.6044[\mathrm{Nm} / \mathrm{rad}]$ \\
$l_{g 2}$ & $0.0743[\mathrm{~m}]$ & & \\
\hline
\end{tabular}

TABLE VI

CONTROL PARAMETERS (EXPERIMENT)

\begin{tabular}{|c|c||c|l||l|l|}
\hline$\theta_{10}$ & $137.3[\mathrm{deg}]$ & $\alpha$ & 6.0 & $v_{\text {rel }}$ & $13.9[\mathrm{~m} / \mathrm{s}]$ \\
$\theta_{20}$ & $-90.0[\mathrm{deg}]$ & $r_{d 1}$ & 0.39 & $\dot{y}_{r e l}$ & $0.0[\mathrm{~m} / \mathrm{s}]$ \\
& & $r_{d 2}$ & 0.39 & & \\
\hline
\end{tabular}

Second, we proposed a control method to realize throwing motion in a desired direction. The control method uses output zeroing to make the ball position converge on the desired path. We obtained throwing motion in the desired direction in each model through numerical simulations. For the spring model, by using optimization for the parameters of the output function, we showed the efficient desired path about energy cost utilizing spring effect. For the constraint model, we showed the advantage of proposed model and control method by comparing with 1-link model. Finally, the experimental result showed the effectiveness of proposed method, but it is still in progress.

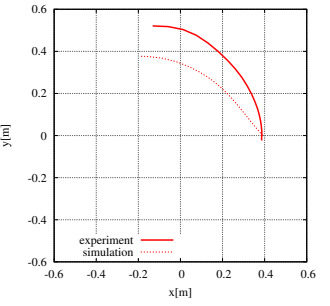

(a) Trajectory of the ball : $x_{b}, y_{b}$

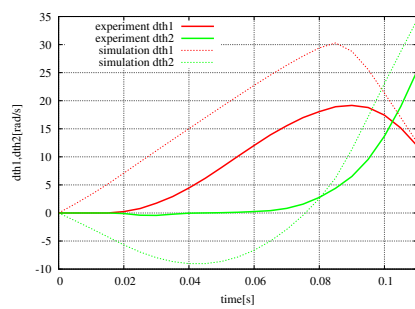

(c) Angular velocity : $\dot{\theta}_{1}, \dot{\theta}_{2}$

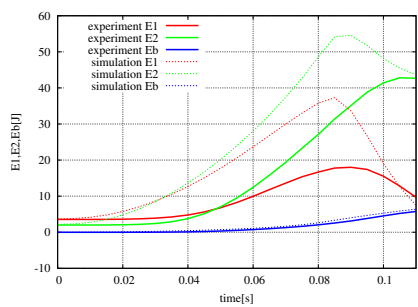

(e) Energy : $E_{1}, E_{2}, E_{b}$

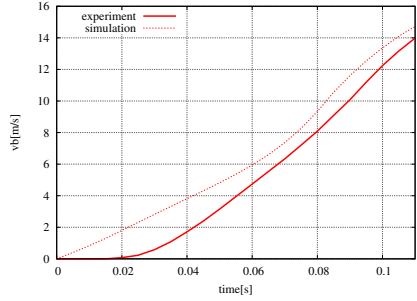

(b) Ball velocity : $v_{b}$

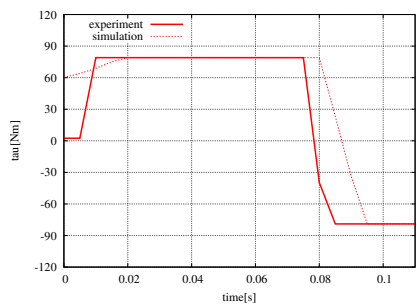

(d) Input torque : $\tau$

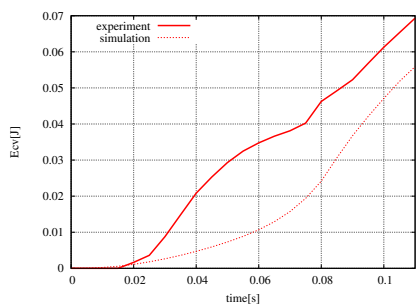

(f) $E_{c v}$
Fig. 17. Throwing experiment (Spring model)

\section{REFERENCES}

[1] Ellen Kreighbaum, Katharine M. Barthels, BIOMECHANICS: A Qualitative Approach for Studying Human Movement, Benjamin-Cummings Pub Co, 1996.

[2] Chunquan Xu, Aiuo Ming, Makoto Shimojo, "Optimal Trajectory Generation for Manipulator with Strong Nonlinear Constraints and Multiple Boundary Conditions", IEEE Int. Conf. on Robotics and Biomimetics, 2004, pp.192-197.

[3] Chunquan Xu, Takeharu Nagaoka, Aiguo Ming, Makoto Shimojo, "Motion Control of Golf Swing Robot Based on Target Dynamics", IEEE Int. Conf. on Intelligent Robots and Systems, 2006, pp.25452550.

[4] Masafumi Okada, Shigeki Ban, Yoshihiko Nakamura, "Skill of Compliance with Controlled Charging/Discharging of Kinetic Energy", IEEE Int. Conf. on Robotics and Automation, vol.3, 2002, pp.24552460.

[5] Senoo T., Namiki A., Ishikawa M., "High-speed batting using a multijointed manipulator", IEEE Int. Conf. on Robotics and Automation vol.2, 2004, pp.1191-1196.

[6] Hitoshi Arisumi, Tetuo Kotoku, Kiyoshi Komoriya, "A Study of Casting Manipulation(Swing Motion Control and Planning Throwing Motion)", IEEE Int. Conf. on Intelligent Robots and Systems, vol.1, 1997, pp.168-174.

[7] Kato N.,Matsuda K.,Nakamura T., "Adaptive Control for a Throwing Motion of a 2 DOF Robot", Int. Workshop on Advanced Motion Control, vol.1, 1996, pp.203-207.

[8] Minor M.A., Jensen K., Youngshik Kim, "Design and control of a three-link serial manipulator for lessons in particle dynamics", IEEE Int. Conf. on Robotics and Automation, vol.4, 2002, pp.3435-3441.

[9] Taku Senoo, Akio Namiki, Masatoshi Ishikawa, "High-speed Throwing Motion Based on Wave Propagation", JSME Conf. on Robotics and Mechatronics(in Japanese), 2007.

[10] Alberto Isidori, Nonlinear Control Systems, Springer 1995. 